University of South Carolina

Scholar Commons

$12-1-2008$

\title{
Predicting Young's Modulus of Nanowires from First-Principles Calculations on their Surface and Bulk Materials
}

\author{
Guofeng Wang \\ Xiaodong Li \\ University of South Carolina - Columbia, lixiao@cec.sc.edu
}

Follow this and additional works at: https://scholarcommons.sc.edu/emec_facpub

Part of the Applied Mechanics Commons, and the Other Mechanical Engineering Commons

\section{Publication Info}

Published in Journal of Applied Physics, Volume 104, Issue 11, 2008, pages \#113517-.

(C) Journal of Applied Physics 2008, American Institute of Physics.

-Wang, G. \& Li, X. (1 December 2008). Predicting Young's Modulus of Nanowires from First-Principles Calculations on their Surface and Bulk Materials. Journal of Applied Physics, 104 (11), \#113517.

http://dx.doi.org/10.1063/1.3033634

This Article is brought to you by the Mechanical Engineering, Department of at Scholar Commons. It has been accepted for inclusion in Faculty Publications by an authorized administrator of Scholar Commons. For more information, please contact digres@mailbox.sc.edu. 


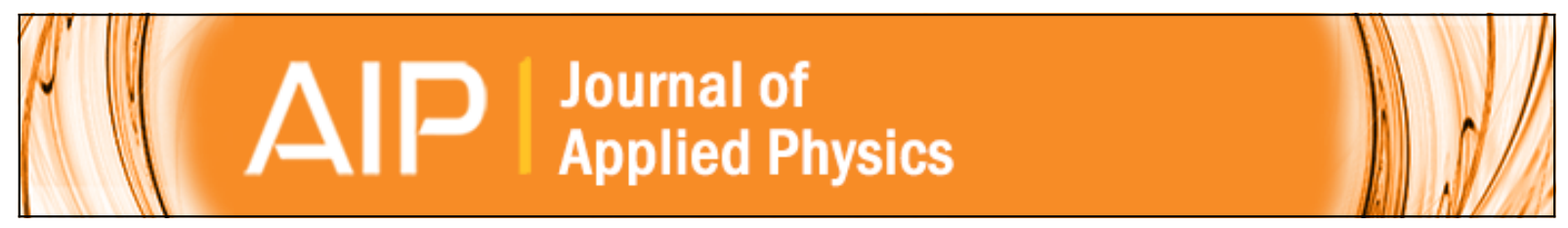

Predicting Young's modulus of nanowires from first-principles calculations on their surface and bulk materials

Guofeng Wang and Xiaodong Li

Citation: Journal of Applied Physics 104, 113517 (2008); doi: 10.1063/1.3033634

View online: http://dx.doi.org/10.1063/1.3033634

View Table of Contents: http://scitation.aip.org/content/aip/journal/jap/104/11?ver=pdfcov

Published by the AIP Publishing

\section{Articles you may be interested in}

First principles investigations on the electronic structure of anchor groups on $\mathrm{ZnO}$ nanowires and surfaces

J. Appl. Phys. 115, 203720 (2014); 10.1063/1.4879676

Surface elasticity effect on the size-dependent elastic property of nanowires

J. Appl. Phys. 111, 083506 (2012); 10.1063/1.3703671

Size effects on formation energies and electronic structures of oxygen and zinc vacancies in $\mathrm{ZnO}$ nanowires: A first-principles study

J. Appl. Phys. 109, 044306 (2011); 10.1063/1.3549131

Magnetic coupling properties of Mn-doped $\mathrm{ZnO}$ nanowires: First-principles calculations

J. Appl. Phys. 103, 073903 (2008); 10.1063/1.2903332

Influence of electromechanical boundary conditions on elasticity of zinc oxide nanowires

Appl. Phys. Lett. 91, 183106 (2007); 10.1063/1.2805027
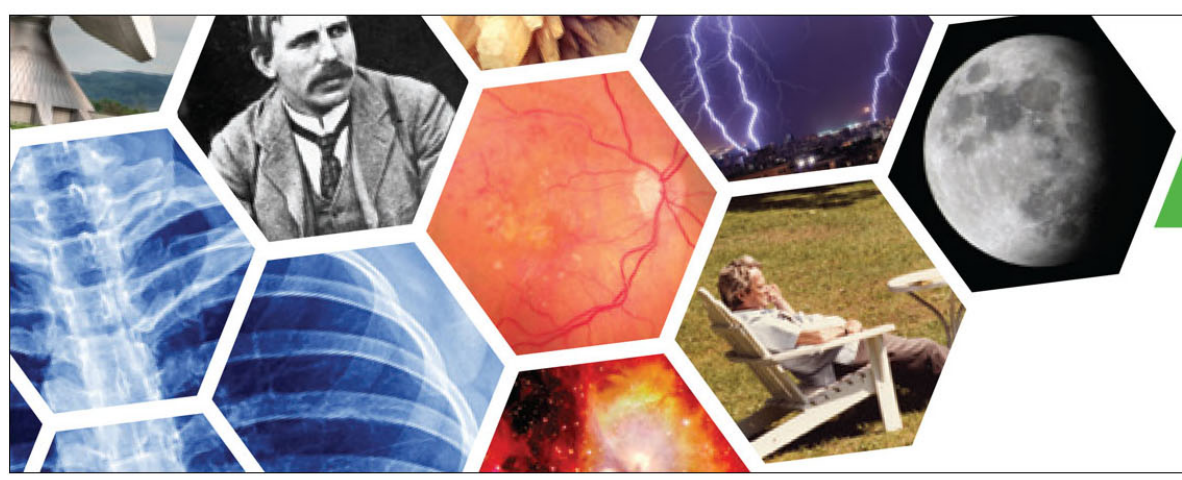

\section{SUBSCRIBE TO \\ physics today}




\title{
Predicting Young's modulus of nanowires from first-principles calculations on their surface and bulk materials
}

\author{
Guofeng Wang ${ }^{1, \mathrm{a})}$ and Xiaodong $\mathrm{Li}^{2}$ \\ ${ }^{1}$ Department of Mechanical Engineering, Indiana University-Purdue University Indianapolis, \\ Indianapolis, Indiana 46202, USA \\ ${ }^{2}$ Department of Mechanical Engineering, University of South Carolina, Columbia, \\ South Carolina 29208, USA
}

(Received 14 July 2008; accepted 17 October 2008; published online 8 December 2008)

\begin{abstract}
Using the concept of surface stress, we developed a model that is able to predict Young's modulus of nanowires as a function of nanowire diameters from the calculated properties of their surface and bulk materials. We took both equilibrium strain effect and surface stress effect into consideration to account for the geometric size influence on the elastic properties of nanowires. In this work, we combined first-principles density functional theory calculations of material properties with linear elasticity theory of clamped-end three-point bending. Furthermore, we applied this computational approach to $\mathrm{Ag}, \mathrm{Au}$, and $\mathrm{ZnO}$ nanowires. For both $\mathrm{Ag}$ and $\mathrm{Au}$ nanowires, our theoretical predictions agree well with the experimental data in the literature. For $\mathrm{ZnO}$ nanowires, our predictions are qualitatively consistent with some of experimental data for $\mathrm{ZnO}$ nanostructures. Consequently, we found that surface stress plays a very important role in determining Young's modulus of nanowires. Our finding suggests that the elastic properties of nanowires could be possibly engineered by altering the surface stress of their lateral surfaces. (C) 2008 American Institute of Physics.
\end{abstract}

[DOI: $10.1063 / 1.3033634]$

\section{INTRODUCTION}

Currently, many powerful electromechanical devices can be designed and fabricated at the microlevel such as microelectromechanical systems. It is a highly active research forefront in developing future electromechanical devices with size in the nanoscale, for instance, nanoelectromechanical systems (NEMSs). ${ }^{1}$ For example, a nanogenerator, which uses $\mathrm{ZnO}$ nanowires to convert mechanical energy to electrical energy, has been successfully fabicated. ${ }^{2}$ NEMS employs one-dimensional (1D) nanomaterials (nanowires, nanobelts, and/or nanotubes) as its active components to generate, transmit, and convert powers and motions. Hence, the dependence of the mechanical properties on the geometric size of 1D nanomaterials is a very important factor affecting the performance of those 1D nanomaterials in the NEMS devices. So far, several different fashions of the size dependence of the elastic properties of nanomaterials have been revealed: (1) Young's modulus increases with the decreasing size, for example, in Ag and Pd nanowires; ${ }^{3,4}$ (2) Young's modulus decreases with the decreasing size, for example, in $\mathrm{Cr}$ and Si nanocantilevers; ${ }^{5,6}$ and (3) Young's modulus shows little dependence on the size of the nanomaterials such as $\mathrm{Au}$ nanowires. ${ }^{7}$ Moreover, for the same nanomaterial, different research groups could observe and report the opposite size dependence of its elastic properties. For the case of $1 \mathrm{D} \mathrm{ZnO}$ nanomaterials, some measurements ${ }^{8-13}$ indicated that their Young's modulus should be lower than the bulk modulus. In contrast, some investigations ${ }^{14,15}$ declared the observation of the exact opposite trend.

\footnotetext{
a) Author to whom correspondence should be addressed. Electronic mail: wang83@iupui.edu.
}

Despite its significance, the mechanism for how Young's modulus of 1D nanomaterials depends on their size and surface conditions is barely understood. Generally speaking, the surface of the nanomaterials will exert great influence on its overall mechanical properties. Consequently, Young's modulus of a 1D nanomaterial is believed to be determined by both bulk elastic modulus and surface elastic modulus. ${ }^{16,17} \mathrm{In}$ other words, the elastic response of a 1D nanomaterial depends strongly on its surface elastic constant. ${ }^{18}$ The relative increase or decrease in the elastic properties of nanomaterials may be the result of surface bonding ${ }^{19}$ and bulk nonlinear phenomena. ${ }^{20}$ Therefore, it is of great interest to quantitatively investigate how to predict the elastic properties of $1 \mathrm{D}$ nanomaterials from the properties of the surface and bulk materials. In this paper, we will present a model developed for that purpose.

Our model mainly focuses on the role of surface stress effect in determining Young's modulus of 1D nanomaterials. Surface stress is defined as the reversible work per unit area required to elastically stretch a surface. ${ }^{21}$ The experimental data and analysis in Ref. 3 showed that a positive (tensile) surface stress will lead to an increase in Young's modulus as the diameter decreases. Our previous theoretical study pointed out that a negative (compressive) surface stress is the reason for the observed decrease in Young's modulus with decreasing diameter of the $\mathrm{ZnO}$ nanowires. ${ }^{22}$ Recently, it was proposed that the surface stress effect is also responsible for the reduction in the resonant frequencies of fixed/fixed $\mathrm{Si}$ nanowires $^{23}$ and the asymmetric yield strength of $\mathrm{Al}$ nanowires. ${ }^{24}$ Here, we further elucidate how the surface stress affects Young's modulus of nanowires.

This paper is structured as follows: in Sec. II, we present our model that predicts Young's modulus of the nanowire 
with a given diameter based on surface stress effect. In Sec. III, we give the required properties of bulk and surface materials calculated using first-principles density functional theory (DFT) method and then evaluate Young's modulus of the nanowires using our developed model. Our model predictions are compared with the available experimental data in this section; at last, final conclusions are drawn in Sec. IV.

\section{MODELS}

In this work, we derived the quantitative relation between Young's modulus of a nanowire (with a circular cross section) and its material properties assuming clamped-end three-point bending loading condition. The total energy of such nanowire is expressed as the sum of the energy contributions from bulk and surface materials. We assumed that the external surface of nanowires is a surface layer with a thickness $t$,

$$
U=\frac{\pi(D-2 t)^{2}}{4} L \Omega(\varepsilon)+\pi D L \gamma(\varepsilon),
$$

where $\Omega(\varepsilon)$ is the bulk energy density in the nanowire core, $\gamma(\varepsilon)$ is the surface energy of the nanowire surfaces, $D$ is the diameter of the nanowire, and $L$ is the length of the nanowire.

\section{A. Effect of equilibrium strain}

The equilibrium bulk material has the lowest energy at the zero-strain state of the crystal lattices. However, the lowest-energy configuration of the surface layers does not necessarily have the same lattice parameters as the zerostrain state of the bulk materials. This is because the arrangement and separation of atoms are different in the surface and in the bulk crystal. Hence, the bulk energy density $\Omega(\varepsilon)$ and the surface energy $\gamma(\varepsilon)$ can be written in the following forms:

$$
\begin{aligned}
& \Omega(\varepsilon)=\Omega_{\min }+\frac{1}{2} K_{b} \varepsilon^{2}, \\
& \gamma(\varepsilon)=\gamma_{\min }+\frac{1}{2} K_{s}\left(\varepsilon-\varepsilon_{0}^{S}\right)^{2},
\end{aligned}
$$

where $K_{b}$ is the bulk elastic modulus, $K_{s}$ is related to the elastic modulus of infinitely large extended surface, and $\varepsilon_{0}^{S}$ is the strain at which the surface energy reaches its minimum. $\Omega_{\min }$ and $\gamma_{\min }$ are the minimal bulk energy density and the minimal surface energy, respectively.

Thus, we could determine the equilibrium strain $\left(\varepsilon^{*}\right)$ in the nanowire through $(\partial U / \partial \varepsilon)=0$ at $\varepsilon^{*}$ by considering Eqs. (1) and (2),

$$
\varepsilon^{*}=\frac{K_{s} \varepsilon_{0}^{S}}{\frac{(D-2 t)^{2}}{4 D} K_{b}+K_{s}} .
$$

The existence of this equilibrium strain in the nanowires has already been revealed in previous molecular dynamics simulations. $^{25,26}$ The equilibrium strain would affect the elastic modulus of the core (bulk) region of the nanowires.
If a nanowire is deformed by a strain $\delta$ from its equilibrium state (with an equilibrium strain $\varepsilon^{*}$ ) under a load, then $\delta=\varepsilon /\left(1+\varepsilon^{*}\right)$ (note that $\varepsilon$ is the strain with respect to the equilibrium crystal lattice). Hence, Young's modulus contributed from the nanowire's core should be

$$
E_{\text {core }}=\frac{\partial^{2} \Omega(\varepsilon)}{\partial \delta^{2}}=\left(1+\varepsilon^{*}\right)^{2} E_{b}
$$

\section{B. Effect of surface stress}

Surface stress $(g=\gamma+\partial \gamma / \partial \varepsilon)$ is the reversible work per unit area required to elastically stretch a surface. ${ }^{21}$ When a circular nanowire is subject to a deformation, its total surface area will change by $\pi D(1-\nu) \Delta L$ ( $\nu$ is Poisson's ratio). Consequently, the energy change associated with the surface deformation of the nanowire is $\Delta U_{S}=\pi D(1-\nu) g \Delta L$. It is noted that the change in nanowire length $(\Delta L)$ is dependent upon the loading conditions: for axial tensile loading, $\Delta L$ is linearly proportional to the strain. In contrast, $\Delta L$ is proportional to the square of the deflection under clamped-end three-point bending.

Clamped-end three-point bending test is most often used to measure Young's modulus of nanostructures. ${ }^{4,11}$ In this work, we derive our model just for this loading condition. When a concentrated load $P$ acts at the middle point of a nanowire with a length of $L$ and fixed on both ends, the elastic deflection curve of the nanowire would be as follows: ${ }^{27}$

$$
y(x)=\frac{P x^{2}}{48 E I}(3 L-4 x) \quad\left(0 \leq x \leq \frac{L}{2}\right) .
$$

For the nanowire with a circular cross section, moment of inertia $I=\pi D^{4} / 64$. Thus, the maximum deflection is $d$ $=P L^{3} / 192 E I$ occurring at $x=L / 2$. In experimental tests, Young's modulus of the nanowire is determined by $E$ $=(P / d)\left(L^{3} / 192 I\right)$. by

The elongation of the nanowire under bending is given

$$
\Delta L=\int_{0}^{L / 2}\left[y^{\prime}(x)\right]^{2} d x=\frac{12}{5} \frac{d^{2}}{L} .
$$

Consequently, the resistant force to the bending due to surface stress in the nanowire is

$$
P_{S}=-\frac{\partial U_{S}}{\partial d}=-\frac{24}{5} \pi D(1-\nu) g \frac{d}{L} .
$$

Hence, Young's modulus contributed from the nanowire's surface should be

$$
E_{\text {surface }}=\left(\frac{-P_{S}}{d}\right)\left(\frac{L^{3}}{192 I}\right)=\frac{8}{5} g(1-\nu) \frac{L^{2}}{D^{3}} .
$$

Similar derivation was done previously by Cuenot et $a l^{3}$ Note that $E_{\text {surface }}$ in Eq. (8) is Young's modulus of the curved circular nanowire surface, different from $K_{s}$ in Eqs. (2b) and (3) referring to the elastic modulus of the flat extended surface along a specific crystalline direction. 
TABLE I. Calculated equilibrium lattice constants $(a)$, elastic constants $\left(C_{11}, C_{12}\right.$, and $\left.C_{44}\right)$, Young's moduli $(E)$, and Poisson's ratios $(\nu)$ of fcc Ag and Au using first-principles DFT method. For comparison, the experimental values are also given.

\begin{tabular}{lcccc}
\hline \hline & \multicolumn{2}{c}{$\mathrm{Ag}$} & \multicolumn{2}{c}{$\mathrm{Au}$} \\
\cline { 2 - 5 } & This work & Expt. & This work & Expt. \\
\hline$a(\AA)$ & 4.159 & $4.09^{\mathrm{a}}$ & 4.174 & $4.08^{\mathrm{a}}$ \\
$C_{11}(\mathrm{GPa})$ & 117.7 & $124^{\mathrm{b}}$ & 165.9 & $186^{\mathrm{b}}$ \\
$C_{12}(\mathrm{GPa})$ & 90.3 & $93.4^{\mathrm{b}}$ & 142.2 & $157^{\mathrm{b}}$ \\
$C_{44}(\mathrm{GPa})$ & 38.0 & $46.1^{\mathrm{b}}$ & 26.7 & $42^{\mathrm{b}}$ \\
$E(\mathrm{GPa})$ & $77.5^{\mathrm{c}}$ & $79^{\mathrm{d}}$ & $59.5^{\mathrm{c}}$ & $80^{\mathrm{d}}$ \\
$\nu$ & $0.370^{\mathrm{e}}$ & $0.38^{\mathrm{d}}$ & $0.434^{\mathrm{e}}$ & $0.42^{\mathrm{d}}$ \\
\hline
\end{tabular}

${ }^{a}$ Reference 34.

${ }^{\mathrm{b}}$ Reference 35.

${ }^{\mathrm{c}} E=\left(C_{11}-C_{12}+3 C_{44}\right)\left(C_{11}+2 C_{12}\right) /\left(2 C_{11}+3 C_{12}+C_{44}\right)$.

${ }^{\mathrm{d}}$ Reference 36.

${ }^{\mathrm{e}} \nu=5\left(C_{11}+2 C_{12}\right) /\left(4 C_{11}+6 C_{12}+2 C_{44}\right)-1$.

\section{Young's modulus of nanowire}

Combining Eqs. (4) and (8), we express Young's modulus of nanowires, which have circular cross sections and are subject to clamped-end three-point bending, as the following function:

$$
E_{\text {nanowire }}=\left(1+\varepsilon^{*}\right)^{2} E_{b}+\frac{8}{5} g(1-\nu) \frac{L^{2}}{D^{3}} .
$$

With the increase in the diameter $(D)$ of the nanowires, the equilibrium strain $\varepsilon^{*}$ would approach zero according to Eq. (3). Therefore, $E_{\text {nanowire }}$ in Eq. (9) would be equal to bulk Young's modulus $E_{b}$ when $D$ reaches the limit of bulk materials.

\section{RESULTS}

In order to examine the reliability of Eq. (9), we performed first-principles calculations on example materials $\mathrm{Ag}$, $\mathrm{Au}$, and $\mathrm{ZnO}$, predicted their size-dependent Young's moduli based on Eq. (9) using the calculated theoretical data, and further compared our predictions with experimental measurements. Our first-principles calculations for the three materials were performed using the VASP code. ${ }^{28,29}$ We used the projector augmented wave method ${ }^{30}$ and the generalized gradient approximation of Perdew and Wang ${ }^{31}$ for exchange and correlation. We chose a kinetic energy cutoff of $600 \mathrm{eV}$ to expand the electronic wave functions in the plane wave basis. The energy convergence for all geometry optimization was set to be $1 \times 10^{-5} \mathrm{eV}$.

\section{A. Bulk materials}

For $\mathrm{Ag}, \mathrm{Au}$, and $\mathrm{ZnO}$ in their bulk states, we computed the equilibrium lattice constants and the corresponding elastic constants using first-principles calculations. The results are given in Tables I and II. The equilibrium $\mathrm{Ag}$ and $\mathrm{Au}$ crystals have face-centered cubic (fcc) structures, while equilibrium $\mathrm{ZnO}$ has a wurtzite crystal structure. Through calculations, we are able to determine the lattice constants that lead to the minimal energy of the respective crystal structures. Using $16 \times 16 \times 16 k$-point grid for $k$-space integra-
TABLE II. Calculated equilibrium lattice constants ( $a$ and $c$ ), internal parameter $(u)$, elastic constants $\left(C_{11}, C_{12}, C_{13}, C_{33}\right.$, and $\left.C_{55}\right)$, Young's modulus $(E)$, and Poisson's ratio $(\nu)$ of wurtzite $\mathrm{ZnO}$ using first-principles DFT method. For comparison, the experimental values are also given.

\begin{tabular}{lcc}
\hline \hline & This work & Expt. \\
\hline$a(\AA)$ & 3.282 & $3.2496^{\mathrm{a}}$ \\
$c(\AA)$ & 5.292 & $5.2042^{\mathrm{a}}$ \\
$u$ & 0.380 & $0.3819^{\mathrm{b}}$ \\
$C_{11}(\mathrm{GPa})$ & 191.7 & $209.7^{\mathrm{c}}$ \\
$C_{12}(\mathrm{GPa})$ & 110.0 & $121.1^{\mathrm{c}}$ \\
$C_{13}(\mathrm{GPa})$ & 96.7 & $105.1^{\mathrm{c}}$ \\
$C_{33}(\mathrm{GPa})$ & 203.4 & $210.9^{\mathrm{c}}$ \\
$C_{55}(\mathrm{GPa})$ & 37.2 & $42.5^{\mathrm{c}}$ \\
$E(\mathrm{GPa})$ & $113.8^{\mathrm{d}}$ & $111.2^{\mathrm{e}}$ \\
$\nu$ & $0.357^{\mathrm{d}}$ & $\cdots$ \\
\hline \hline
\end{tabular}

${ }^{\mathrm{a}}$ Reference 37.

${ }^{\mathrm{b}}$ Reference 38 .

${ }^{\mathrm{c}}$ Reference 39.

${ }^{\mathrm{d}} E=(F-G+3 H)(F+2 G) /(2 F+3 G+H)$ and $\nu=5(F+2 G) /(4 F+6 G+2 H)$ $-1 ;$ here, $\quad F=\left(2 C_{11}+C_{33}\right) / 3, \quad G=\left(C_{12}+2 C_{13}\right) / 3, \quad$ and $H=\left(4 C_{44}+C_{11}\right.$ $\left.-C_{12}\right) / 6$.

${ }^{\mathrm{e}}$ Reference 40.

tion, we determined the elastic constants of the crystals following the procedure given in Ref. 32 (for fcc $\mathrm{Ag}$ and $\mathrm{Au}$ ) and Ref. 33 (for wurtzite $\mathrm{ZnO}$ ). As shown in Tables I and II, our theoretical predictions agree well with the corresponding experimental data. Furthermore, we calculated the averaged bulk Young's modulus and Poisson's ratio from our theoretical results of the elastic constants. These two elastic properties of bulk materials are needed in Eq. (9) to predict Young's modulus of nanowires.

\section{B. Surface materials}

For fcc $\mathrm{Ag}$ and $\mathrm{Au}$, the (111) surface has the lowest surface energy and thus is the most probable external surfaces of their nanostructures. For $\mathrm{ZnO}$ nanowires grown along [0001] direction, $(10 \overline{1} 0)$ and $(11 \overline{2} 0)$ surfaces are their lateral facets. Consequently, we conducted the DFT relaxation calculations for the (111) surface slab of fcc Ag (and $\mathrm{Au})$ and $(10 \overline{1} 0)$ and $(11 \overline{2} 0)$ surface slabs of wurtzite $\mathrm{ZnO}$. In our surface calculations, we used a surface slab (with two surfaces) with $N_{s}$ layers of atoms in a periodic supercell which contains $N_{b}$ atomic layers in the direction normal to the surface. To simulate the (111) surface of fcc $\mathrm{Ag}$ and $\mathrm{Au}$, we used a supercell spanned with three orthogonal directions

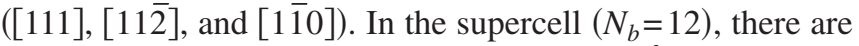
seven layers of atoms $\left[N_{s}=7\right.$, about $2 t=14.4 \AA$ thick for the $\mathrm{Ag}$ (111) slab and $2 t=14.5 \AA$ thick for the Au (111) slab] and five layers of vacuum. For the (111) surface calculations, we used $8 \times 12 \times 2 k$-point grid for $k$-space integration. For (1010) surfaces, we used a supercell $\left(N_{b}=16\right)$ containing eight atom layers $\left(N_{s}=8\right.$, about $2 t=9.5 \AA$ thick $)$ and eight layers of vacuum. For $(11 \overline{2} 0)$ surface, we used a supercell $\left(N_{b}=12\right)$ containing six atom layers $\left(N_{s}=6\right.$, about $2 t$ $=8.2 \AA$ thick) and six layers of vacuum. For $k$-space integration, we used a $10 \times 6 \times 2 k$-point grid for $(10 \overline{10})$ surface and a $6 \times 6 \times 2 k$-point grid for $(11 \overline{2} 0)$ surface. 
Using slabs to simulate surfaces, we need to conciliate two requirements: (a) the slab should be thin enough to represent two-dimensional surfaces and (b) the two surfaces of the slab should be separated far enough to minimize their interactions. In this work, we made our above choice of the thickness of the slabs by examining the separation of the layers in the slab after relaxation. For seven-layer Ag and $\mathrm{Au}$ (111) slabs, the separation of the central layers differs by less than $0.2 \%$ from the corresponding bulk value. For eightlayer $\mathrm{ZnO}(10 \overline{1} 0)$ slab, the separation of the central layers differs by $5 \%$ from the corresponding bulk value in contrast the separation of the outermost surface layers differs by $49.8 \%$ from the corresponding bulk value. For six-layer $\mathrm{ZnO}$ $(11 \overline{2} 0)$ slab, the separation of the central layers differs by $3 \%$ from the corresponding bulk value, while the separation of the outermost surface layers differs by $18.1 \%$ from the corresponding bulk value. In this way, we attained a slab model whose central region is very close to the bulk materials and whose surface regions are subjected to the relaxation in vacuum.

Surface relaxation is quite insignificant for the (111) surfaces of $\mathrm{Ag}$ and $\mathrm{Au}$. After geometry optimization, the outermost surface layer is found to move inward by $0.3 \%$ of its bulk layer separation for Ag. This is close to the experimental measurement of about $0.0 \%$ relaxation of $\mathrm{Ag}$ (111) surface. ${ }^{41}$ For $\mathrm{Au}$, we found that the outermost surface layer moves outward by $1.2 \%$ of its bulk layer separation. Prior DFT calculations also predicted an outward motion of the outermost layer of $\mathrm{Au}$ (111) surface. $^{42}$

However, significant relaxations in both $(10 \overline{1} 0)$ and $(11 \overline{2} 0)$ surfaces of $\mathrm{ZnO}$ have been observed from our calcu-

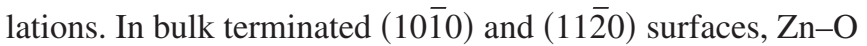
dimers lay parallel to the surface. In contrast, the $\mathrm{Zn}-\mathrm{O}$ dimers would tilt relative to the surface after relaxation. We found in our calculations that in the outermost layer of (1010) surface, $\mathrm{Zn}$ cations move inward by $\Delta d_{\perp}(\mathrm{Zn})=$ $-0.34 \AA$ and move laterally toward $\mathrm{O}$ by $\Delta d_{\|}(\mathrm{Zn})=0.16 \AA$, $\mathrm{O}$ anions move inward by $\Delta d_{\perp}(\mathrm{O})=-0.002 \AA$, and the resultant tilt angle of $\mathrm{Zn}-\mathrm{O}$ dimers to be $\omega=10.6^{\circ}$. Our results for the (1010) surface are in good agreement with the experimental data ${ }^{43} \quad\left[\Delta d_{\perp}(\mathrm{Zn})=-0.45 \pm 0.1 \AA, \quad \Delta d_{\|}(\mathrm{Zn})\right.$ $=0.1 \pm 0.2 \AA, \quad \Delta d_{\perp}(\mathrm{O})=-0.05 \pm 0.1 \AA$, and $\left.\omega=12 \pm 5^{\circ}\right]$. We also found in the outermost layer of (1120) surface the tilt angle for $\mathrm{Zn}-\mathrm{O}$ dimers to be $7.7^{\circ}$ and a $6.1 \%$ reduction in the $\mathrm{Zn}-\mathrm{O}$ bond length, consistent with previous DFT results. ${ }^{44}$

Furthermore, we calculated the surface energy $(\gamma)$ and surface stress tensor $(g)$ for the relaxed $\mathrm{Ag}, \mathrm{Au}$, and $\mathrm{ZnO}$ surfaces using Eq. (10) as suggested in Ref. 45,

$$
\begin{aligned}
& \gamma=\frac{1}{2 A}\left[E_{s}-\left(N_{s} / N_{b}\right) E_{b}\right], \\
& g_{\alpha \beta}=\frac{1}{2 A}\left[V \sigma_{\alpha \beta}^{s}-\left(N_{s} / N_{b}\right) V_{b} \sigma_{\alpha \beta}^{b}\right],
\end{aligned}
$$

where $A$ is the area on one side of the surface slab; $E_{s}, V$, and $\sigma_{\alpha \beta}^{s}$ are the energy, volume, and stress tensor for the super-
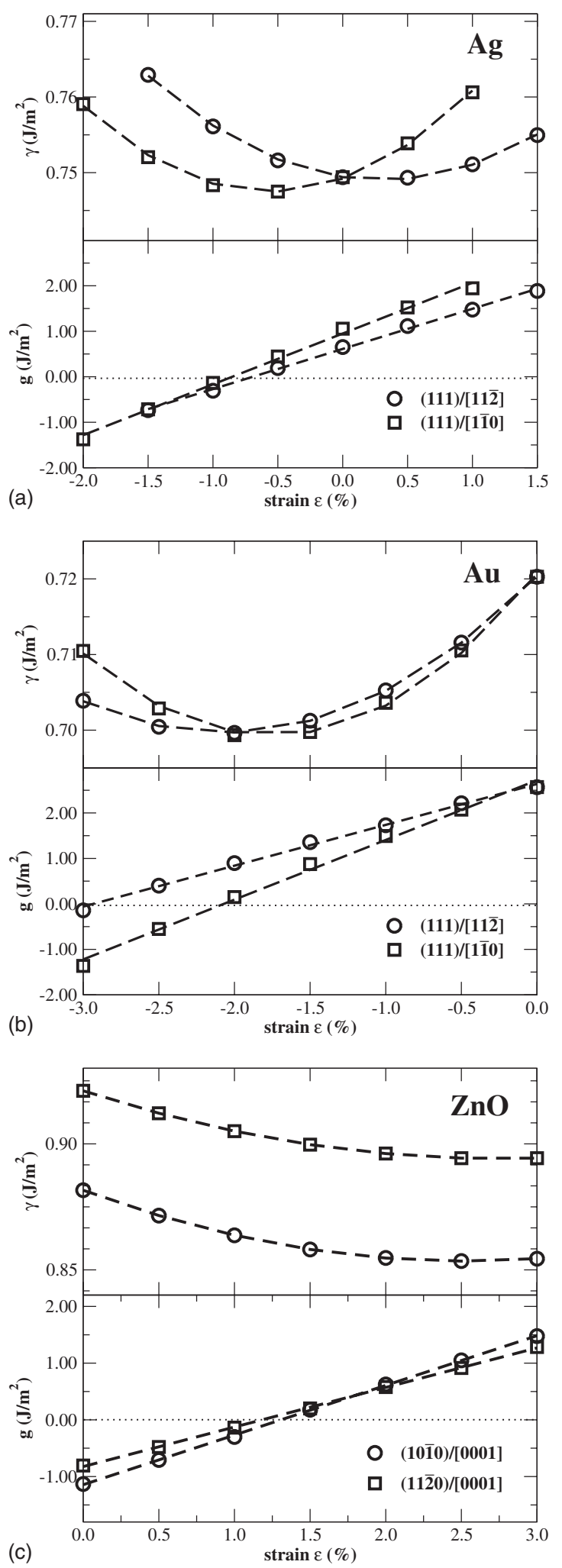

FIG. 1. Variation in the surface energies $(\gamma)$ and the surface stresses $(g)$ along (a) the $[11 \overline{2}]$ and $[1 \overline{1} 0]$ directions of $\mathrm{Ag}(111)$ surface, (b) the [11 $\overline{2}]$ and [1 10$]$ directions of $\mathrm{Au}$ (111) surface, and (c) the [0001] direction of $\mathrm{ZnO}(10 \overline{10})$ and $(11 \overline{2} 0)$ surfaces. In the figures, the dashed lines in the upper panel are the quadratic function fitting of calculated surface energy data and the dashed lines in the lower panel are the linear fitting of calculated surface stress data.

cell containing the relaxed surface slab; and $E_{b}, V_{b}$, and $\sigma_{\alpha \beta}^{b}$ are the energy, volume, and stress tensor of the equilibrium bulk materials in the supercell. 
To examine strain effect, we deformed the surfaces by a strain of $\varepsilon$, relaxed the surface slabs at each given strain, and calculated the corresponding surface energy and surface stress using Eq. (10). In Fig. 1, we plot the calculated surface energies and surface stresses as a function of strain $\varepsilon$ for $\mathrm{Ag}$, $\mathrm{Au}$, and $\mathrm{ZnO}$. For $\mathrm{Ag}$ and $\mathrm{Au}$, we deformed the (111) surfaces along both $[11 \overline{2}]$ and $[1 \overline{1} 0]$ directions. For $\mathrm{ZnO}$, we expanded the (1010) and (1120) surfaces along the [0001] direction.

Figure 1(a) shows that the surface energy of $\mathrm{Ag}$ (111) varies with the strain in a quadratic function form. The surface energy will have its minimum value at a strain of $0.3 \%$

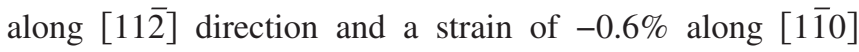
direction. Based on Eq. (3), the equilibrium strain in the $\mathrm{Ag}$ nanowires would be larger than $-0.6 \%$. The surface stress exhibits a linear function of strain and is always positive when the strain in the surface is larger than $-0.7 \%$. Hence, surface stress will enhance (or stiffen) Young's modulus of $\mathrm{Ag}$ nanowires according to Eq. (9). It is noticeable in the lower panel of Fig. 1 that the calculated surface stresses along $[11 \overline{2}]$ and $[1 \overline{1} 0]$ directions are not equal to each other at the zero-strain state of $\mathrm{Ag}$ (111) surface. This result is quite puzzling because the surface stress should be isotropic for the undeformed (111) surface of fcc crystals owing to its threefold rotational symmetry. ${ }^{25}$ In some further tests, we found that a non-negligible discrepancy in the calculated surface stress along the two directions still exists even after we increase the $k$-points for integration, vary the number of surface layers in our model, and/or switch to ultrasoft pseudopotential. Consequently, it appears that there is some numerical error in determining surface stresses using DFT method. However, our results (Fig. 3) will show that such errors in the values of calculated surface stress do not have much effect on our model prediction of Young's modulus of $\mathrm{Ag}$ nanowires.

Figure 1(b) for $\mathrm{Au}$ (111) surface indicates that the minimum of its surface energy will be at a strain of $-2.1 \%$ along $[11 \overline{2}]$ direction and a strain of $-1.8 \%$ along $[1 \overline{1} 0]$ direction. The surface stress would change from negative to positive at the strain of $-2.9 \%$ along the $[11 \overline{2}]$ direction and the strain of $-2.1 \%$ along the [1ㅣㅣ direction. Conceivably, surface stress will enhance Young's modulus of Au nanowires.

In stark contrast to the results of $\mathrm{Ag}$ and $\mathrm{Au}$, it is seen in Fig. 1(c) for the two surfaces of wurtzite $\mathrm{ZnO}$ that the surface energies decrease with the expansion strain up to about $2.5 \%$, while the surface stresses (along [0001] direction) increase with the strain and change signs (from negative to positive) at the strain about $1.25 \%$. Thus, our results in Fig. 1(c) qualitatively point out that along [0001] direction the nonpolar surfaces with a strain below $1.25 \%$ would facilitate elastic deformation and decrease (or soften) the elastic modulus of $1 \mathrm{D} \mathrm{ZnO}$ nanomaterials.

\section{Model predictions}

Figures 1(b) and 1(c) show clearly that the surfaces do not always have their minimum-energy configuration at the state with a zero strain, where the bulk materials would reach

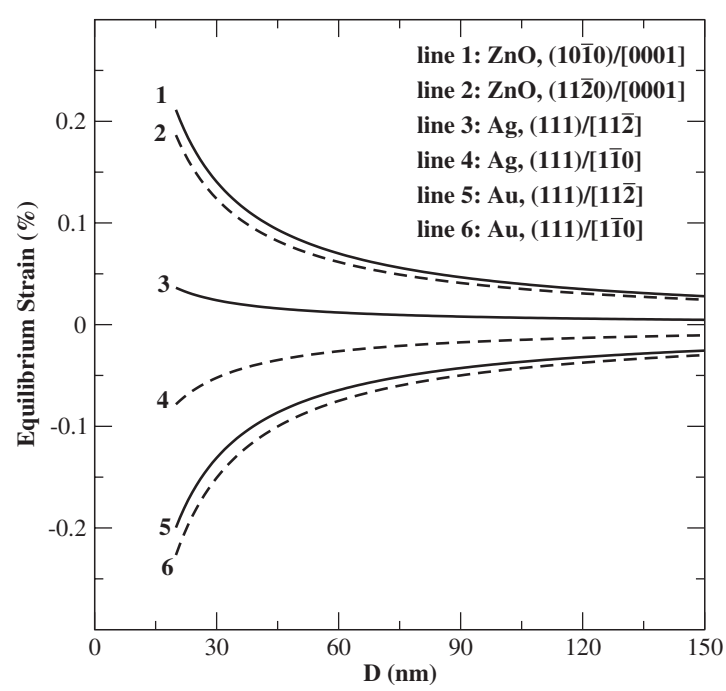

FIG. 2. Calculated equilibrium strains in the equilibrium $\mathrm{Ag}, \mathrm{Au}$, and $\mathrm{ZnO}$ nanowires as a function of nanowire diameter.

their minimum energy. Thus, the surfaces will exert a contraction (for $\mathrm{Au}$ ) or expansion (for $\mathrm{ZnO}$ ) force on the whole nanowires. As a result, it is expected that the equilibrium $\mathrm{Au}$ nanowires are shorter than their bulk material counterparts and the equilibrium $\mathrm{ZnO}$ nanowires are longer than their bulk material counterparts. To quantify this equilibrium strain, we calculated the energy variations with the strains for bulk materials using DFT method. Then, we employed Eq. (3) to find the equilibrium strain $\left(\varepsilon^{*}\right)$ for the nanowires with different diameters. We plotted the calculated equilibrium strain compared to the bulk lattice parameters in Fig. 2 for $\mathrm{Ag}, \mathrm{Au}$, and $\mathrm{ZnO}$. Using the surface energies of $(10 \overline{1} 0) /[0001]$ and $(11 \overline{2} 0) /[0001]$ in Fig. 1(c), we predict an appreciable elongation (positive equilibrium strain) in the equilibrium $\mathrm{ZnO}$ nanowires. Using the surface energies of $(111) /[11 \overline{2}]$ and $(111) /[1 \overline{1} 0]$ in Fig. 1(b), we predict an appreciable contraction (negative equilibrium strain) in the equilibrium Au nanowires. However, we found in the equilibrium Ag nanowires a small positive strain using the surface energies of $(111) /[11 \overline{2}]$ [Fig. 1(a)] but a small negative strain using the surface energies of (111)/[1] $\overline{1} 0]$ [Fig. 1(a)].

Through first-principles calculations, we have determined the value of bulk Young's modulus $\left(E_{b}\right)$, Poisson's ratio $(\nu)$, equilibrium strain $\left(\varepsilon^{*}\right)$, and surface stress $(g)$ as a function of strain. In this work, we assume $L=1000 \mathrm{~nm}$, which is the typical suspended length of the nanowires in atomic force microscopy three-point bending tests. Inputting all those calculated material-dependent parameters into Eq. (9), we predict Young's moduli of the nanowires for Ag (Fig. 3), Au (Fig. 4), and $\mathrm{ZnO}$ (Fig. 5) as functions of their diameters.

In Fig. 3, we predict that Young's modulus of Ag nanowire increases when its diameter decreases. This is because $\mathrm{Ag}$ (111) surfaces have a positive surface stress and thus enhance Young's modulus of the nanowires. Our theoretical predictions (lines) agree excellently with experimental measurement data (circles) in Fig. 3. Moreover, our model predicts in Fig. 4 that Young's modulus of Au nanowire would 


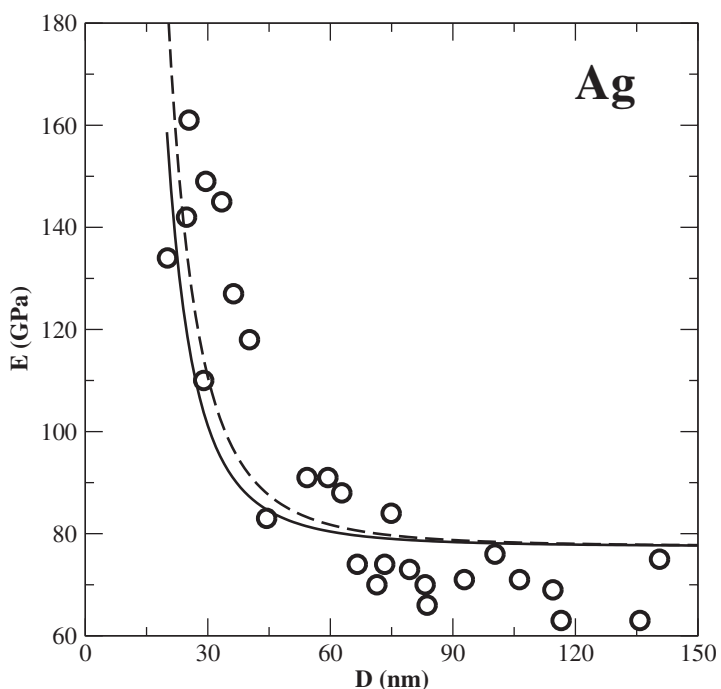

FIG. 3. Size dependency of Young's modulus of Ag nanowires enclosed by (111) surfaces. The solid line and dashed line show the model predictions using the surface properties of $(111) /[11 \overline{2}]$ and $(111) /[1 \overline{1} 0]$, respectively. For comparison, the experimental data from Ref. 4 are plotted as circles.

also increase when its diameter decreases due to its positive surface stress. Although it was believed that Young's modulus of Au nanowires is "independent" of diameter in Ref. 7, Fig. 4 shows that the same experimental data (especially the data for the Au nanowires with diameters around $50 \mathrm{~nm}$ ) are actually consistent with our model of enhancing Young's modulus for Au nanowires.

It is worth mentioning that the increase in Young's modulus with the decreasing diameter of Au nanowires is quite slow when the nanowire diameters are larger than 75 $\mathrm{nm}$. The calculated Young's modulus of the Au nanowire with a diameter of $75 \mathrm{~nm}$ is only about $9 \%$ higher than the bulk Young's modulus of $\mathrm{Au}$. This apparent "independency" is a result of two effects: (1) a compressive (negative) equi-

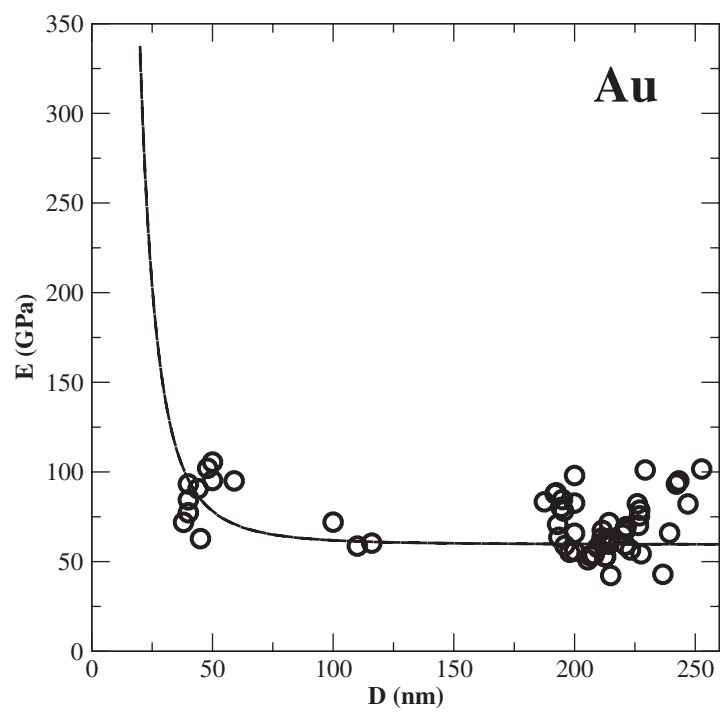

FIG. 4. Size dependency of Young's modulus of Au nanowires enclosed by (111) surfaces. The two dashed lines (overlapped with each other) show the model predictions using the surface properties of $(111) /[11 \overline{2}]$ and $(111) /[1 \overline{1} 0]$. For comparison, the experimental data from Ref. 7 are plotted as circles.

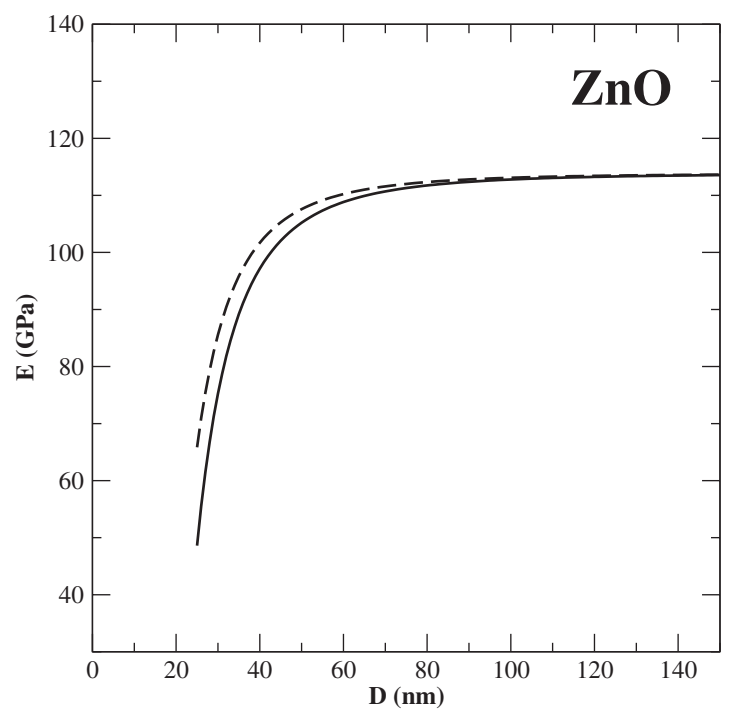

FIG. 5. Predicted size dependency of Young's modulus of $\mathrm{ZnO}$ nanowires enclosed by $(10 \overline{1} 0)$ surfaces (solid line) or (11 $\overline{2} 0)$ surfaces (dashed line).

librium strain in the nanowire core leads to a decrease in Young's modulus of the nanowires, and (2) a positive surface stress in the nanowire surface leads to an increase in Young's modulus of the nanowires. When the diameter of Au nanowires is small (less than $75 \mathrm{~nm}$ ), surface stress effect becomes dominant and is responsible for the sharp increase in Young's modulus as shown in Fig. 4.

Plotted in Fig. 5 for $\mathrm{ZnO}$ nanowires with $D>20 \mathrm{~nm}$, our model predicts a decrease in their Young's modulus when reducing their diameters. This is due to the negative surface stress of $\mathrm{ZnO}$ surfaces [see Fig. 1(c)]. At this moment, there are no available (three-point bending) experimental data of Young's modulus of $\mathrm{ZnO}$ nanowires for us to directly compare our theoretical predictions with. However, there is much experimental evidence that $\mathrm{ZnO}$ nanostructures have a lower elastic modulus than $\mathrm{ZnO}$ bulk materials. ${ }^{8-13}$ Thus, our model prediction in Fig. 5 is qualitatively consistent with those measurements. We also notice that some studies report that $\mathrm{ZnO}$ nanowires have higher Young's modulus that increases with the decreasing size. ${ }^{14,15}$ Here, we postulate an explanation to this discrepancy in the literature. Our results in Figs. 1(c) and 5 are obtained from the fully relaxed $\mathrm{ZnO}$ surfaces, which are quite different from the corresponding bulk terminated surfaces (see details in Sec. III B). We found from our calculations for $\mathrm{ZnO}$ that the surface stresses are negative for the fully relaxed surfaces but positive for the unrelaxed bulk terminated surfaces. This suggests that the value (positive or negative) of surface stress may strongly depend on the extent of the surface relaxation process. Surface relaxation process can be changed by surface contamination or surface charges ${ }^{46}$ which can be introduced during sample preparation and measurement process. Thus, Young's modulus of $\mathrm{ZnO}$ nanowires with the same diameter might exhibit different values due to the surface stress effect for various surface conditions of the experimental samples.

Although our model [Eq. (9)] leads to satisfactory agreement between the theoretical and experimental data for nanowires with a diameter larger than $20 \mathrm{~nm}$, some further im- 
provement is required to accurately predict Young's modulus of small nanowires with a diameter below $20 \mathrm{~nm}$. For example, we express the total energy of nanowires as a linear combination of its bulk and surface contributions in Eq. (1). When the nanowires have very small diameters, some nonlinear term must be included to that equation. In addition, we currently use a flat, extended surface slab to model the nanowire surfaces. For small nanowires, their surfaces are highly curved and hence have a significant transverse stress (strain) component. Thus, the curvature effect must also be properly addressed in an elaborated model for small nanowires.

\section{CONCLUSIONS}

In this work, we first developed a model to predict Young's modulus of nanowires from the properties of the corresponding bulk and surface materials. We included two effects (equilibrium strain and surface stress) in our model to account for the geometric size influence on the elastic properties of nanowires. To accurately describe the surface stress effect, we must know the exact deformation process of nanowires. This is because different loading conditions (for example, axial tensile test and three-point bending test) would lead to different values of the changes in the length and surface area of the nanowires during the elastic deformation. For a direct comparison to experimental data, we focus on studying the clamped-end three-point bending loading condition.

Furthermore, we calculated the bulk and surface properties of the materials using first-principles DFT method. The calculated properties include Young's modulus, Poisson's ratio, surface energy, and surface stress. Taking those theoretical data as inputs, we predicted Young's moduli of $\mathrm{Ag}, \mathrm{Au}$, and $\mathrm{ZnO}$ nanowires as functions of nanowire diameter. To validate our model and approach, we compared our theoretical results with the experimental measurement results. It is found that our model predictions for $\mathrm{Ag}$ and $\mathrm{Au}$ nanowires agree excellently with those experimental data. For $\mathrm{ZnO}$ nanowires with $D>20 \mathrm{~nm}$, our predictions also agree qualitatively with a series of experimental results. We believe that the agreement between our model and experimental data is remarkable since we did not introduce any empirical data modifications in our theoretical approach.

Our model reveals two major effects that the surface exerts on the elastic deformation process of nanomaterials. First, the surface may have different minimum-energy lattice parameters from the bulk lattice parameters of the material and thus results in an equilibrium strain in the nanowire core region. Second, the surface may have tensile or compressive surface stress that is the energy required to elastically deform the surface. A tensile surface stress would lead to an increase in Young's modulus with the decreasing size of nanowires, while a compressive surface stress would lead to a decrease in Young's modulus with the decreasing size of nanowires.

Our model indicates that the tensile (positive) surface stress is the reason for $\mathrm{Ag}$ and $\mathrm{Au}$ nanowires showing an enhanced Young's modulus when reducing nanowire diameters. This viewpoint has been accepted owing to the support of myriads of experimental and simulation studies. ${ }^{4,7,25}$ Our model also points out that the compressive (negative) surface stress in $\mathrm{ZnO}$ nanowires (with $D>20 \mathrm{~nm}$ ) would lead to a softened Young's modulus when reducing nanowire diameters. We notice that some other materials, such as $\mathrm{Si}^{6}{ }^{6}$ $\mathrm{GaN}^{47-49}$ and $\mathrm{ZnS},{ }^{50,51}$ exhibit reduced Young's modulus when decreasing the size of their nanomaterials. Hence, we proposed based on our results that the compressive surface stress, which is an inherent material property, is responsible for the observed lower Young's modulus of those nanomaterials compared to their bulk modulus. Since surface stress plays a very important role in determining Young's modulus of nanomaterials, it is reasonable to expect that the elastic properties of nanomaterials could be engineered by altering the surface stress through rational control of the adsorptions, charges, structure, and impurities in the surfaces.

\section{ACKNOWLEDGMENTS}

The authors thank Dr. G. Y. Jing for providing the experimental Young's modulus data of Ag nanowires and Dr. B. Wu for providing the experimental Young's modulus data of $\mathrm{Au}$ nanowires. G.W. acknowledges the research grants from Richard G. Lugar Center for Renewable Energy and the Office of the Vice Chancellor for Research (RSFG program) at Indiana University-Purdue University Indianapolis. X.L. acknowledges the research grants from the National Science Foundation (Grant Nos. EPS-0296165 and CMMI-0653651), the ACS Petroleum Research Fund (ACS PRF Grant No. 40450-AC10), and the University of South Carolina NanoCenter.

${ }^{1}$ K. L. Ekinci and M. L. Roukes, Rev. Sci. Instrum. 76, 061101 (2005).

${ }^{2}$ X. Wang, J. Song, J. Liu, and Z. L. Wang, Science 316, 102 (2007).

${ }^{3}$ S. Cuenot, C. Frétigny, S. Demoustier-Champagne, and B. Nysten, Phys. Rev. B 69, 165410 (2004).

${ }^{4}$ G. Y. Jing, H. L. Duan, X. M. Sun, Z. S. Zhang, J. Xu, Y. D. Li, J. X. Wang, and D. P. Yu, Phys. Rev. B 73, 235409 (2006).

${ }^{5}$ X. Li, T. Ono, Y. Wang, and M. Esashi, Appl. Phys. Lett. 83, 3081 (2003).

${ }^{6}$ S. G. Nilsson, X. Borrisé, and L. Montelius, Appl. Phys. Lett. 85, 3555 (2004).

${ }^{7}$ B. Wu, A. Heidelberg, and J. J. Boland, Nature Mater. 4, 525 (2005).

${ }^{8}$ X. D. Bai, P. X. Gao, Z. L. Wang, and E. G. Wang, Appl. Phys. Lett. 82, 4806 (2003).

${ }^{9}$ J. Song, X. Wang, E. Riedo, and Z. L. Wang, Nano Lett. 5, 1954 (2005).

${ }^{10}$ M. H. Zhao, C. B. Jiang, S. X. Li, and S. X. Mao, Mater. Sci. Eng., A 409, 223 (2005).

${ }^{11}$ H. Ni and X. D. Li, Nanotechnology 17, 3591 (2006).

${ }^{12}$ W. Mai and Z. L. Wang, Appl. Phys. Lett. 89, 073112 (2006).

${ }^{13}$ M. Lucas, W. Mai, R. Yang, Z. L. Wang, and E. Reido, Nano Lett. 7, 1314 (2007).

${ }^{14}$ C. Q. Chen, Y. Shi, Y. S. Zhang, J. Zhu, and Y. J. Yan, Phys. Rev. Lett. 96, 075505 (2006).

${ }^{15}$ G. Stan, C. V. Ciobanu, P. M. Parthangal, and R. F. Cook, Nano Lett. 7, 3691 (2007).

${ }^{16}$ J. Q. Broughton, C. A. Meli, P. Vashishta, and R. K. Kalia, Phys. Rev. B 56, 611 (1997).

${ }^{17}$ R. E. Miller and V. B. Shenoy, Nanotechnology 11, 139 (2000).

${ }^{18}$ V. B. Shenoy, Phys. Rev. B 71, 094104 (2005).

${ }^{19}$ L. G. Zhou and H. C. Huang, Appl. Phys. Lett. 84, 1940 (2004).

${ }^{20}$ H. Y. Liang, M. Upmanyu, and H. C. Huang, Phys. Rev. B 71, 241403 (2005).

${ }^{21}$ R. C. Cammarata, Prog. Surf. Sci. 46, 1 (1994).

${ }^{22}$ G. Wang and X. D. Li, Appl. Phys. Lett. 91, 231912 (2007).

${ }^{23}$ H. S. Park, J. Appl. Phys. 103, 123504 (2008).

${ }^{24}$ W. Zhang, T. Wang, and X. Chen, J. Appl. Phys. 103, 123527 (2008).

${ }^{25}$ J. Diao, K. Gall, and M. L. Dunn, J. Mech. Phys. Solids 52, 1935 (2004).

${ }^{26}$ B. Lee and R. E. Rudd, Phys. Rev. B 75, 041305(R) (2007). 
${ }^{27}$ J. M. Gere and S. P. Timoshenko, Mechanics of Materials (PWS Engineering, Boston, 1987), p. 434.

${ }^{28}$ G. Kresse and J. Hafner, Phys. Rev. B 47, 558 (1993).

${ }^{29}$ G. Kresse and J. Furthmüller, Phys. Rev. B 54, 11169 (1996).

${ }^{30}$ G. Kresse and D. Joubert, Phys. Rev. B 59, 1758 (1999).

${ }^{31}$ J. P. Perdew and Y. Wang, Phys. Rev. B 45, 13244 (1992).

${ }^{32}$ P. Söderlind, O. Eriksson, J. M. Wills, and A. M. Boring, Phys. Rev. B 48, 5844 (1993).

${ }^{33}$ L. Fast, J. M. Wills, B. Johansson, and O. Eriksson, Phys. Rev. B 51, 17431 (1995).

${ }^{34}$ N. W. Ashcroft and N. D. Mermin, Solid State Physics (Holt, Rinehart and Winston, New York, 1976).

${ }^{35}$ G. Simmons and H. Wang, Single Crystal Elastic Constants and Calculated Aggregate Properties: A Handbook (MIT, Cambridge, 1971).

${ }^{36} \mathrm{H}$. Rolnick, Phys. Rev. 36, 506 (1930).

${ }^{37}$ H. Karzel, W. Potzel, M. Köfferlein, W. Schiessl, M. Steiner, U. Hiller, G. M. Kalvius, D. W. Mitchell, T. P. Das, P. Blaha, K. Schwarz, and M. P. Pasternak, Phys. Rev. B 53, 11425 (1996).

${ }^{38}$ J. Albertsson, S. C. Abrahams, and Å. Kvick, Acta Crystallogr. B 45, 34 (1989).

${ }^{39}$ T. B. Bateman, J. Appl. Phys. 33, 3309 (1962).
${ }^{40}$ S. O. Kucheyev, J. E. Bradby, J. S. Williams, C. Jagadish, and M. V. Swain, Appl. Phys. Lett. 80, 956 (2002).

${ }^{41}$ F. Soria, J. L. Sacedon, P. M. Echenigire, and D. Titherington, Surf. Sci. 68, 448 (1977)

${ }^{42}$ Ž. Crljen, P. Lazić, D. Šokčević, and R. Brako, Phys. Rev. B 68, 195411 (2003).

${ }^{43}$ C. B. Duke, R. J. Meyer, A. Paton, and P. Mark, Phys. Rev. B 18, 4225 (1978).

${ }^{44}$ B. Meyer and D. Marx, Phys. Rev. B 67, 035403 (2003).

${ }^{45}$ R. J. Needs, Phys. Rev. Lett. 58, 53 (1987).

${ }^{46}$ G. A. Somorjai, Annu. Rev. Phys. Chem. 45, 721 (1994).

${ }^{47}$ C. Y. Nam, P. Jaroenapibal, D. Tham, D. E. Luzzi, S. Evoy, and J. E. Fischer, Nano Lett. 6, 153 (2006).

${ }^{48}$ H. Ni, X. D. Li, G. S. Cheng, and R. Klie, J. Mater. Res. 21, 2882 (2006).

${ }^{49}$ G. Feng, W. D. Nix, Y. Yoon, and C. J. Lee, J. Appl. Phys. 99, 074304 (2006).

${ }^{50}$ X. D. Li, X. X. Wang, Q. H. Xiong, and P. C. Eklund, Nano Lett. 5, 1982 (2005).

${ }^{51}$ Q. Xiong, N. Duarte, S. Tadigadapa, and P. C. Eklund, Nano Lett. 6, 1904 (2006). 